\title{
Performance Enhancement of the Micromixer by the Multiobjective Genetic Algorithm and Surrogate Model Based on a Navier-Stokes Analysis Using Trade-Off Objective Functions
}

\author{
Shakhawat Hossain $\mathbb{D}^{1},{ }^{1}$ Farzana Islam $\mathbb{D}^{2},{ }^{2}$ Nass Toufik Tayeb, ${ }^{3}$ Muhammad Aslam $\left(\mathbb{D},{ }^{4}\right.$ \\ and Jin-Hyuk Kim $\mathbb{D}^{5}$ \\ ${ }^{1}$ Department of Industrial and Production Engineering, Jashore University of Science and Technology, Jessore 7408, Bangladesh \\ ${ }^{2}$ Department of Nanotechnology and Advanced Materials Engineering, Sejong University, Seoul 05006, Republic of Korea \\ ${ }^{3}$ Gas Turbine Joint Research Team, University of Djelfa, Djelfa 17000, Algeria \\ ${ }^{4}$ Department of Chemical Engineering, COMSATS University Islamabad (CUI), Lahore Campus, Defense Road, \\ Off Raiwind Road, Lahore, Pakistan \\ ${ }^{5}$ Carbon Neutral Technology ReD Department, Korea Institute of Industrial Technology, Cheonan 31056, Republic of Korea
}

Correspondence should be addressed to Jin-Hyuk Kim; jinhyuk@kitech.re.kr

Received 29 March 2021; Revised 3 July 2021; Accepted 26 July 2021; Published 3 August 2021

Academic Editor: Amin Jajarmi

Copyright (c) 2021 Shakhawat Hossain et al. This is an open access article distributed under the Creative Commons Attribution License, which permits unrestricted use, distribution, and reproduction in any medium, provided the original work is properly cited.

\begin{abstract}
Optimal structure of the micromixer with a two-layer serpentine crossing device was accomplished by a multiobjective genetic algorithm and surrogate modeling based on a Navier-Stokes analysis using the trade-off objective functions behavior. The optimization analysis was conducted with three design parameters, i.e., channel width to the pitch span $(w / P)$ ratio, major channel width to the pitch span $(H / P)$ ratio, and channel depth to the pitch span $(d / P)$ ratio. Two objective functions (i.e., mixing index and pressure drop) with trade-off characteristics have been used to solve the multiobjective optimization problem. The design domain was predetermined by a parametric investigation; afterward, the Latin hypercube sampling method was employed to select the appropriate design points surrounded by the design domain. The numerical data of the thirty-two design points were used to create the surrogate model; among the different surrogate models, in this study, the Kriging metamodel has been used. The concave pareto-optimal curve signifies the trade-off characteristics linking the objective functions.
\end{abstract}

\section{Introduction}

Proficient and fast mixing of the liquids is mainly a very difficult task in the enhancement of lab-on-a-chip (LOC) as well as $\mu$-TAS investigations. For the low Reynolds number where the inertia force of liquid is insufficient, the mixing mechanism in the microfluidic system is mostly conducted by the diffusion behavior of fluids as a replacement for of turbulence [1-4]. Furthermore, the diffusion mechanism depends on retention time of fluids that is very sluggish; thus, the process needs longer travel length. To resolve the complexity of the mixing mechanism in the microscale, numerous active mixing mechanisms, such as ultrasonic vibration, electrokinetic instability, and bubble-induced acoustic actuation, were employed. Although the active micromixer provides fast mixing, the mechanism to incorporate with microfluidic components is very complicated. Also, the active device creates difficulty in its production, process, and cleaning. On the other hand, passive devices do not need any power unit, and mixing can occur by the structural variations; therefore, passive devices are very simple to fabricate and amalgamate with the microfluidic system [4-6]. As a result, passive micromixers turn out to be much admired selection in the microfluidic analysis compared to the active micromixers. 
Different commercial softwares have become a very reliable and convenient tool to investigate the fluid flow and mixing performance in microfluidic devices [7-9]. In recent times, various micromixers have been invented to acquire the proficient and fast mixing. In laminar flow regime, the chaotic mechanism behavior created by the cyclic disturbance, with the fluid flow, can evidently develop the mixing mechanisms $[10,11]$. The three-dimensional serpentine device [12] was investigated numerically to construct the chaotic behavior through enlarging and shrinking of the fluid flow streams. To generate the appropriate chaotic behavior, the device requires relatively high Reynolds number greater than 25. The micromixer with slanting grooves at the base floor could produce the twisting flow mechanism studied by Stroock et al. [13]. A chaotic micromixer incorporating with square barriers on the oblique grooves design is investigated by Kim et al. [14], and the chaotic flow behavior of fluids was produced due to the regular perturbation. Researchers [15] observed the mechanism of chaotic flow with staggered double-layer of crisscross microdevices; expanding and crimping of the fluid streams methods are responsible to create the chaotic advection. Two-layer crossing microchannels (TLCCM) were proposed and designed [16]; the experimental and simulation findings represent micromixers could perform very high performance of mixing at $(\operatorname{Re}<0.2)$ low Reynolds number. Recently, the Lattice Boltzmann method (LBM) [17-19] becomes a very popular and advance numerical tool to solve the fluid flow phenomena.

Structural optimization through the CFD method becomes a popular and well-situated tool to enhance the micromixers performance. Structural optimization of a micromixer with slanted groove was performed [9] using the electroosmotic flow mechanism; their study illustrates the enhanced mixing performance. The study also represents that the objective function is very sensitive to grooves angle and grooves depth. Structural optimize of the SHG micromixer [20] was performed using the radial basis neural network (RBNN) with three design parameters. The structural optimization of the micromixer with a pattern grooves microchannel was performed [21] with four different parameters; analysis confirms that the performance of the micromixer was reasonably enhanced with the design parameters. A surrogate model based on the weightedaverage (WTA) technique has been used to determine the optimal geometry [22] of the modified Tesla structure in terms of mixing performance and pressure drop; the two objective functions were merged with a weighting factor to create the single-objective optimization problem. The structural optimization of the staggered herringbone micromixer (SHG) with the pattern grooves microchannel was performed using two different objective functions $[23,24]$, i.e., mixing performance at the exit and pressure drop. Researchers performed $[25,26]$ the double-objective optimization process to find the best match of a micromixer with sinusoidal walls (convergent-divergent) and a structure with Sigma unit. Concave pareto-optimal curves were found to demonstrate the connection between objective functions.
Recently, various researchers performed the development of the different micromixer designs and optimization methodologies. From the above discussion, we can conclude that multiobjective optimization is beneficial to negotiate the optimal finding between the various objective functions. The optimization technique (multiobjective) is a very popular and convenient method for the designer to choose required outfits. The existing work signifies a multiobjective optimization problem of a two-layer serpentine crossing device combined with the multiobjective genetic algorithm (MOGA), Navier-Stokes analysis, and surrogate technique. Two objective functions (i.e., mixing index and pressure drop) with trade-off characteristics have been used to solve the multiobjective optimization problem. The numerical data of the thirty-two design points were used to create the surrogate model; among the different surrogate models, in this study, the Kriging metamodel has been used. The concave pareto-optimal curve signifies the trade-off characteristics linking the objective functions.

\section{Dimensions of the Geometry}

From our preceding work [27], to examine the performance of the micromixer coupled with different layers (upper and lower layer), a microdevice was projected (Figure 1). Inlets were coupled with a crossing (main) microchannel and formed $90^{\circ}$ angle, represented in Figure 1(a). Due to the repeated arrangement of ten mixing segments, the sample fluid streams splits and rejoined repetitively. The dimensions of the device were as follows: major channel width $(H=1.07 \mathrm{~mm})$, channel width $(w=0.15)$, depth of the each channel $(d=0.15 \mathrm{~mm})$, pitch span $(P=0.64)$, vertical segment $(b=0.15)$, and number of mixing units is ten.

\section{Numerical Method}

The investigation of the flow of the sample fluid and mixing performance study was performed through an inclusive CFD software package i.e., ANSYS CFX-15.0 ${ }^{\circledR}$ [28]. The subsequent three-dimensional (3D) continuity (steady) and Navier-Stokes equations were solved analytically to carry the numerical investigations.

Mass equation is as follows:

$$
\nabla \cdot(\rho \vec{V})=0 .
$$

Momentum equation is as follows:

$$
(\vec{V} \cdot \nabla) \vec{V}=-\frac{1}{\rho} \nabla p+\nu \nabla^{2} \vec{V},
$$

where the velocity, density, and kinematic viscosity of the sample fluid were indicated by $V, \rho$, and $v$, correspondingly. The unstructured grid system has been formulated using ANSYS ICEM 15.0. The diffusion problem (which cannot be completely ignored) was optimized using the higher-order numerical method [29]. For each fluid particle, the mass transport equation having steady density and viscosity are an equation of advection-diffusion [30] that can be expressed as 


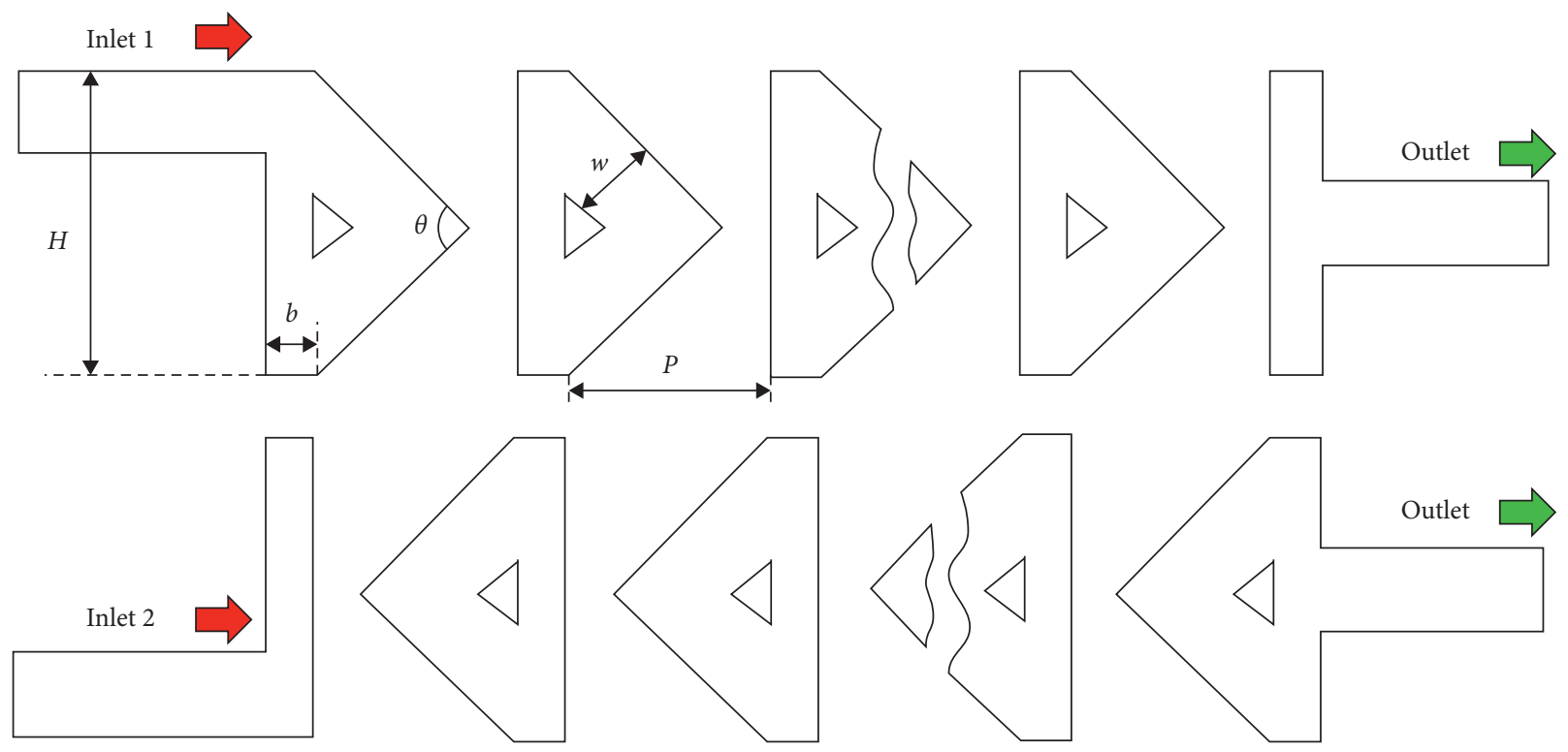

(a)

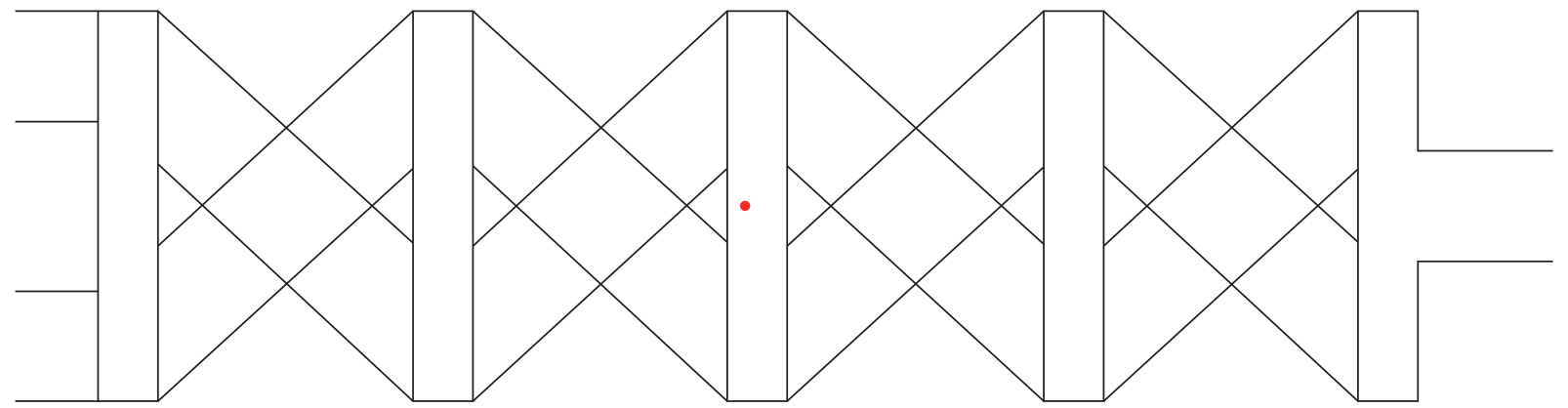

(b)

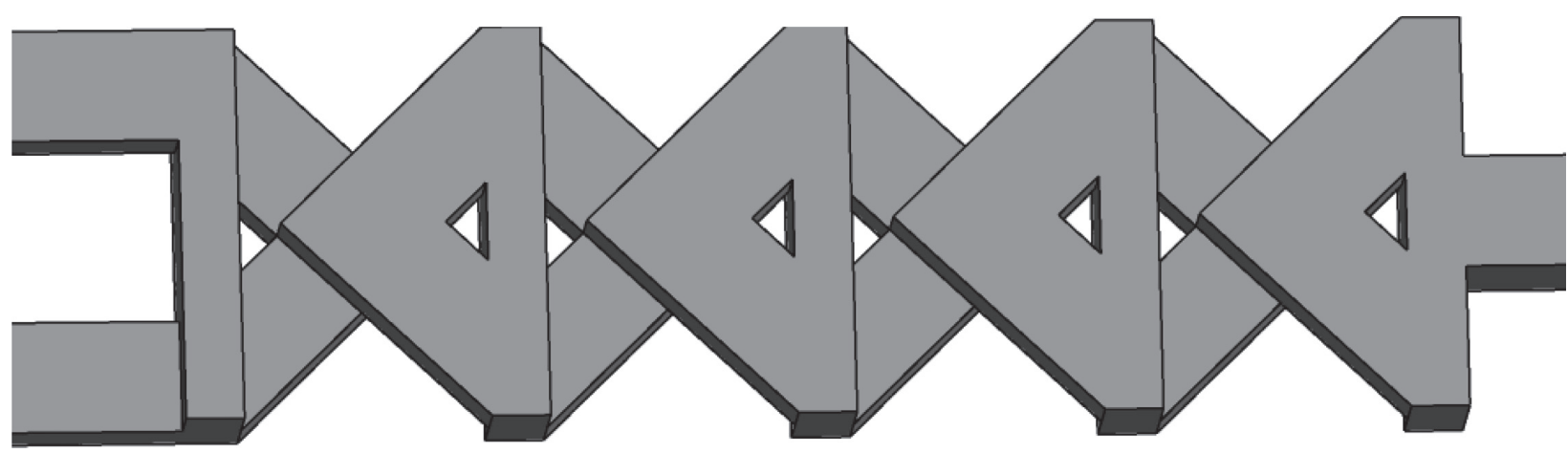

(c)

FIGURE 1: Diagram of the two-layer crossing micromixer [27]. (a) Structural view of upper and bottom layers. (b) Two-dimensional view of the proposed micromixer. (c) Three-dimensional view of the proposed micromixer.

$$
(v \cdot \nabla) C=\alpha \nabla^{2} C,
$$

where the diffusivity coefficient, $\alpha$, and fluid concentration, $C$, were symbolized. For the modeling of the diffusive mixing phenomena, the scalar transport equation has been applied for different micromixers investigation in recent studies and experimentally validated [31-33].

To solve the above equations, subsequent circumstances have been considered. At inlets and outlets, steady velocity of fluid flow and atmospheric pressure were mentioned, correspondingly, and zero speed of the fluid was considered at the walls. Water and dye-water mixture were initiated at inlet 1 and inlet 2, and the mass fraction of water was zero and one, correspondingly. The water was found [16] at $25^{\circ} \mathrm{C}$ as follows: $8.8 \times 10^{-4} \mathrm{~kg} / \mathrm{m}$-s is the dynamic viscosity $(\mu)$ and $997 \mathrm{~kg} / \mathrm{m}^{3}$ is the density $(\rho)$ of the sample fluid [34]. The coefficient of the diffusivity was considered $1 \times 10^{-11} \mathrm{~m}^{2} / \mathrm{s}$ [16] for the dye-water mixture. For the confirmation of the 
highest quality of the results, the governing equation was solved iteratively until the values of the normalized root mean square (RMS) were less than $10^{-8}$. The performance of the micromixer was articulated on a cross-sectional plane along with the flow direction. For an explicit plane, the mass fraction variation of the mixture is expressed as

$$
\sigma=\sqrt{\frac{1}{N} \sum_{i=0}^{N}\left(c_{i}-\bar{c}_{m}\right)^{2}},
$$

where the sampling points on plane are represented by $N$, at point $i$, mass fraction is represented by $c_{i}$, and optimal mass fraction is represented by $c_{m}$. Satisfactory sampling points were measured to confirm the highest quality of the result. For any particular plane, the mixing index is expressed as

$$
M=1-\sqrt{\frac{\sigma^{2}}{\sigma_{\max }^{2}}},
$$

where $\sigma$ and $\sigma_{\max }$ symbolize the deviation of the concentration (standard deviation) at any cross-sectional plane and maximum deviation. The superior value of $M$ indicates better mixing quality, where the mixing index of the sample fluids changes from zero to one. The Reynolds number has been used to approximate the velocity of the sample fluids articulated as follows:

$$
\operatorname{Re}=\frac{\rho V D}{\mu},
$$

where $D$ characterizes the diameter (hydraulic) of the inlet channel.

\section{Design Variables Selection and Objective Functions Determination}

For structural optimization of the micromixer design, the effective parameters have been confirmed through the parametric study. The most important and crucial practice throughout the optimization problem is to pick the proper design boundaries to facilitate the value of objective functions. The involved parametric analysis was examined to pick the sensitive parameters for multiobjective optimization. Three geometric parameters (dimensionless), i.e., channel width to the pitch span $(w / P)$ ratio, major channel width to the pitch span $(H / P)$ ratio, and channel depth to the pitch span $(d / P)$ ratio, were selected to formulate an optimization problem for the projected micromixer. The variety of the design (three) variables was constrained as given in Table 1 . The result of the design parameters (i.e., $H / P, d / P$, and $w / P$ ) on the performance of the mixing index at $\operatorname{Re}=40$ has been studied as shown in Figure 2. Length of the micromixer was constant during investigations. At $\operatorname{Re}=40$, the mixing performance at the outlet of the microdevice is marginally varied with the design parameter $w / P$. The result signifying the deviation of mixing index values was very much sensitive to $H / P(42 \%$ deviation within constrain) than to $d / P(31 \%$ deviation within constrain) and $w / P(25 \%$ deviation within constrain).
TABLE 1: Design variables and their constrains.

\begin{tabular}{lcc}
\hline Design variables & Lower limit & Upper limit \\
\hline$w / P$ & 0.28 & 0.57 \\
$H / P$ & 1.26 & 1.89 \\
$d / P$ & 0.16 & 0.31 \\
\hline
\end{tabular}

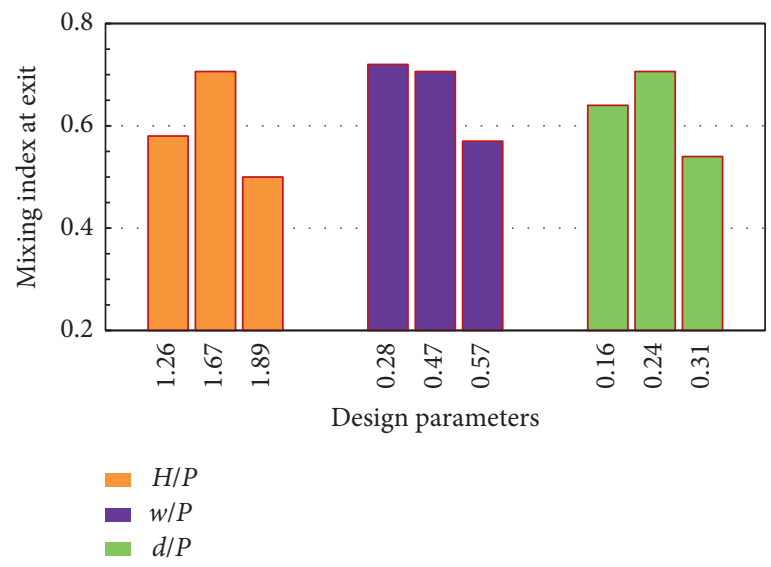

Figure 2: Effect of the design on the mixing index at $\mathrm{Re}=40$.

In this study, the LHS technique was utilized to select the (thirty-two) design points. To examine the trade-off behavior, two objective functions have been considered, namely, mixing enhancement $\left(F_{\mathrm{ME}}\right.$ at $\left.\mathrm{Re}=50\right)$ and overall pressure loss $\left(F_{\Delta P \text { at } \mathrm{Re}=50}\right)$ at Reynolds number 50 . Table 2 provides the reference design with objective functions values. The overall pressure loss is related with the energy required to drive the sample fluids during mixing. Overall pressure loss has been estimated using variation among areaweighted average pressure on a cross-sectional plane to be found at first vertical unit and at the end of the mixing unit.

\section{Surrogate Construction and MOGA}

To formulate the multiobjective optimization problem, the effective design parameters and appropriate design space were chosen cautiously. To compute the values of the objective functions, the 3D Navier-Stokes analysis was used at each design points. The surrogate model has been formulated on the basis of the objective functions values. Surrogate has been formulated to diminish the computational period, as the multiobjective optimization [35] procedures require lots of calculations of the objective functions values in the design space. The flow chart for the multiobjective optimization process to create a global pareto-optimal curve (POF) is shown in Figure 3. The hybrid MOGA has been considered for the solutions of the global pareto-optimal font [36]. Among various surrogate techniques, the Kriging model has been found very suitable match for this work.

This is the deterministic method for the optimization procedure, also nominated as Kriging metamodeling [37]. The model is a function of linear polynomial combined with the Gauss correlation function. The Kriging model mathematically expressed as a pair of the global model and departure can be articulated as 
TABLE 2: Reference design with objective functions values.

\begin{tabular}{|c|c|c|c|c|}
\hline \multicolumn{5}{|c|}{ Reference design } \\
\hline \multicolumn{3}{|c|}{$\begin{array}{l}\text { Design } \\
\text { variables }\end{array}$} & \multicolumn{2}{|c|}{ Objective functions } \\
\hline$w / P$ & $H / P$ & $d / P$ & $\begin{array}{c}\text { Mixing index at } \\
\operatorname{Re}=50\end{array}$ & $\begin{array}{c}\text { Pressure drop }(\mathrm{Pa}) \text { at } \\
\operatorname{Re}=50\end{array}$ \\
\hline 0.47 & 1.67 & 0.24 & 0.69 & $1.32 \times 103$ \\
\hline
\end{tabular}

$$
F(x)=f(x)+Z(x)
$$

where $f(x)$ is the global model, $F(x)$ is the required function which is unknown, and $Z(x)$ represents the localized variation. $Z(x)$ is used to integrate the sampled data points through the Gaussian correlation technique with nonzero and mean zero covariance. The following equation has been used to articulate the covariance matrix:

$$
\operatorname{cov}\left(z\left(x_{i}\right), z\left(x_{j}\right)\right)=\sigma^{2} \exp \left(-\sum_{k=1}^{N} \Theta_{k}\left(x_{i}^{k}-x_{j}^{k}\right)^{2}\right),
$$

where $N$ is the design variables, $\sigma$ is the standard deviation, and $\Theta$ represents the correlation parameter which has been used to construct a relation among the sample data next to the $k$ direction.

\section{Result and Discussion}

The good quality grid system is very much essential to diminish the numerical incorrectness to stimulate using the discretization process. To perform the study, the grid system with tetrahedral mesh used is shown in Figure 4. The grid sensitivity analysis was performed for the enhancement of the mixing index along the microchannel length to determine the suitable number of nodes with the grid system. Grid systems with four different numbers of nodes (i.e., $0.72 \times 10^{6}, 1.42 \times 10^{6}, 1.74 \times 10^{6}$, and $1.89 \times 10^{6}$ ) were executed for the grid dependency test at $\mathrm{Re}=50$ for the reference micromixer (i.e., $w / P=0.47, H / P=1.67$, and $d /$ $P=0.24)$ as shown in Figure 5. A slight difference in mixing performance along the channel length has been visualized for number nodes $1.74 \times 10^{6}$ and $1.89 \times 10^{6}$. Therefore, a grid system having $1.74 \times 10^{6}$ numbers of nodes was preferred as the optimal grid system for further simulations. The numerical model was evaluated qualitatively and quantitatively and with the experimental findings in our earlier article [38].

Mixing index progression is estimated as a function of downway channel length for the micromixer at different Reynolds numbers, i.e., 0.2, 10, 30, 60, and 80 (Figure 6). Succeeding planes (cross-sectional) positioned at every crossing point of the microstructures has been considered to estimate the mixing index. Unluckily, for insufficient inertia energy of sample fluids and the residual period of fluid time, the lowest mixing index was observed at $\operatorname{Re}=10$. Beyond this, Reynolds number coincidentally by strong inertia energy of sample fluids mixing performance enhances significantly as Reynolds numbers increase.

Figure 7 shows the 3D streamlines of the sample fluids to be mixed signifying two various color initiated using each inlets (inlet 1 and inlet 2) at $\operatorname{Re}=10,30$, and 80 were captured to examine the flow configuration which developed the mixing quality. Primarily, sample fluids mixed at the center of the upright segment and go through to the main microchannel. Because of the microchannel construction, the fluid streams are keeping their initial flow passage after the contact of the sample fluid to be mixed. Next, different colored streams came into contact at the very first crossing point. A portion of streamlines is swapped; thus, elongation and thinning of the sample fluids interface occurred at the crossing points which develop the chaotic advection phenomenon. Hence, the two-colored fluid streams are regularly separated into many sublayers throughout a succession of the mixing units. The above mechanism of fluid flow expanded the interfacial segment of flow fluids and reduces the diffusion period through the sample fluids layers; therefore, the method assists the rapid diffusion process and prompt mixing. These processes are rapidly amplified as the Reynolds numbers increases consequently increasing the mixing performance rapidly.

On the basis of the multiobjective optimization algorithm as described, the POFs portentous of the behavior of optimal trade-off involving the pair of (two) contradictory objective functions (mixing enhancement and overall pressure loss) has been obtained. Figure 8 demonstrates the POF for above two objective functions, i.e., $\mathrm{F}_{\mathrm{ME}}$ at $\mathrm{Re}=40$ vs. $\mathrm{F}_{\triangle \mathrm{P}}$ at $\mathrm{Re}=40$. As shown in figure, the concave POF curve corresponds to a considerable development in the mixing index with the increase in the pressure drop. Each solution within the POF is a global pareto-optimal solution; therefore, every objective function has comparable importance within the pareto-optimal solutions. Six different paretooptimal designs (PODs) have been selected carefully on the POF curve to investigate the pareto-optimal solution. At the top extreme end, the POD-1 signifies the maximum mixing performance value with the minimum overall pressure loss; on the other hand, the POD- 6 shows the maximum value of overall pressure loss with the lowest mixing performance value. As mentioned, the behavior of objective functions are paradoxical; thus, the improvement of any objective function (i.e., mixing enhancement) guide to degradation of the other objective functions (i.e., overall pressure loss). The behavior trade-off study demonstrates that maximum mixing performance values could be found at the uppermost overall pressure loss value, while lower overall pressure loss values represent the lower value of the mixing performance. From Figure 8, as contrast to POD-6, POD-1 signifies 38\% comparative improvement in the mixing performance, with $307.4 \%$ improvement in the overall pressure loss.

The accurateness of the optimization algorithm has been evaluated using numerical findings. The numerical solutions of six PODs (i.e., 1, 2, 3, 4, 5, and 6) have been performed as given in Table 3 . Table represents the assessment of objective functions values calculated by numerical simulation and with the surrogate predicted values at Reynolds number 50 . Table also represents the variation in the mixing enhancement at the exit, and overall pressure loss was exaggerated by the design parameters $w / P$ and $d / P$; on the other hand, the other parameter, $H / P$, remains almost constant through the 


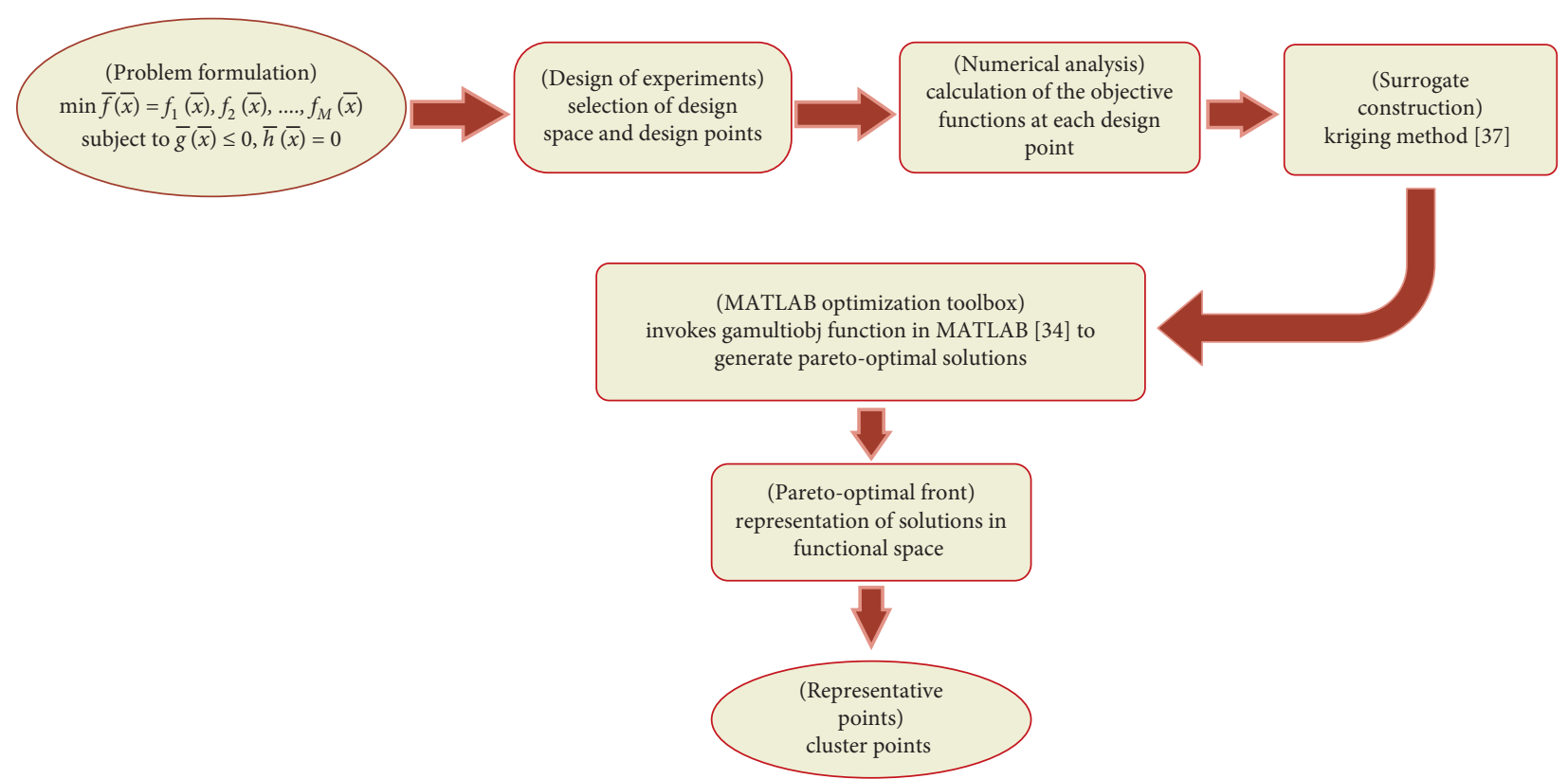

FIGURE 3: Multiobjective optimization algorithm used in this study.

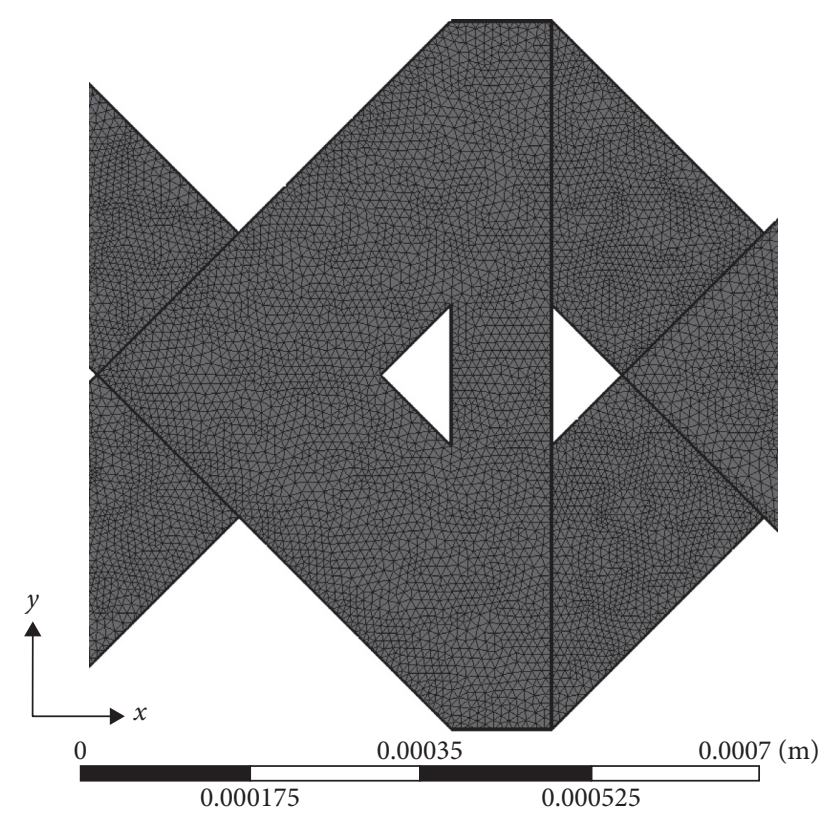

FIgURE 4: Tetrahedral grid system used in this study.

POF. The error proportion enhances as the PODs curve proceeds from top to bottom. Table also corresponds to a good match (between $3.66 \%$ and $10.0 \%$ ) connecting the numerical analysis values and surrogate predicted values.

Figures 9(a) and 9(b) represent the plot of velocity vectors and local vorticity deviations on the planes perpendicular to the flow direction $(y-z)$ at different PODs (i.e., POD-1 and POD-6), respectively. The plane was taken at the end of the last unit. Two reverse revolving vortices have been observed in each $y-z$ planes. The micromixer with POD-1 shows couple of tiny circular-shaped (reverse revolving) vortices packed within the whole plane; on the other hand,

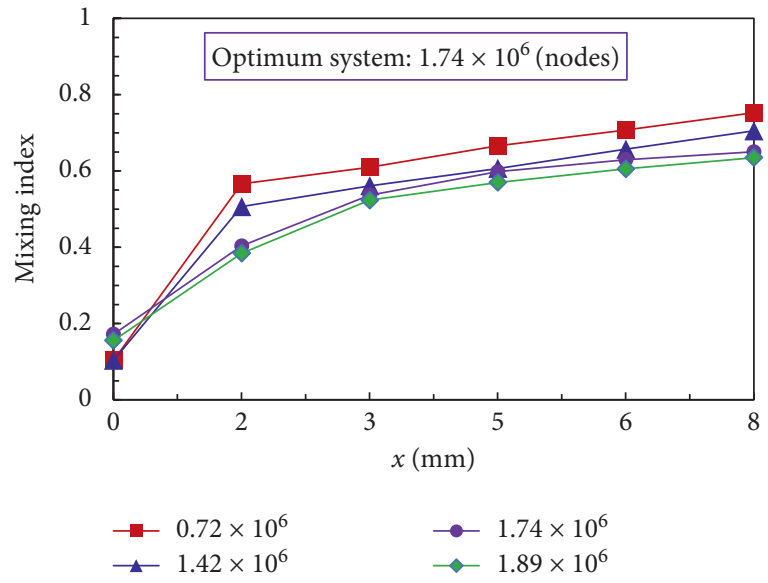

FIgURE 5: Examination of grid dependency for reference design at $\operatorname{Re}=50$.

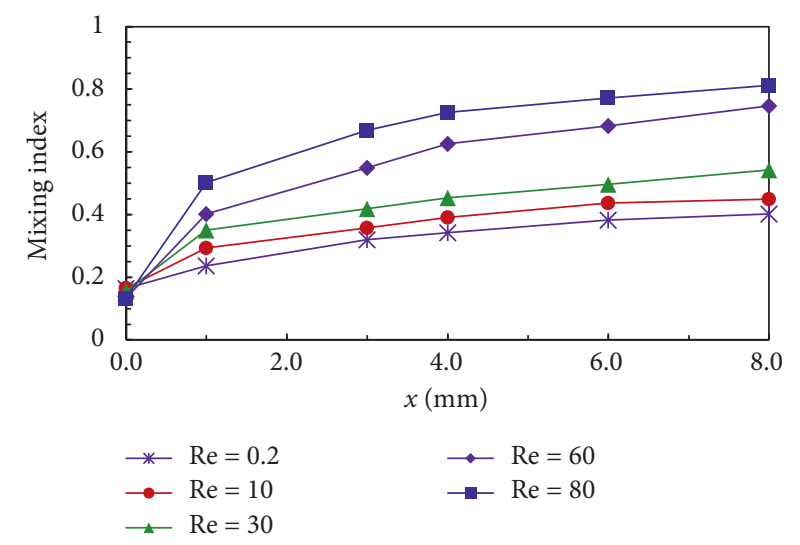

FIGURE 6: Variation of the mixing enhancement through the length of the microchannel at five different Reynolds numbers. $(\operatorname{Re}=0.2$, $10,30,60$, and 80 ). 


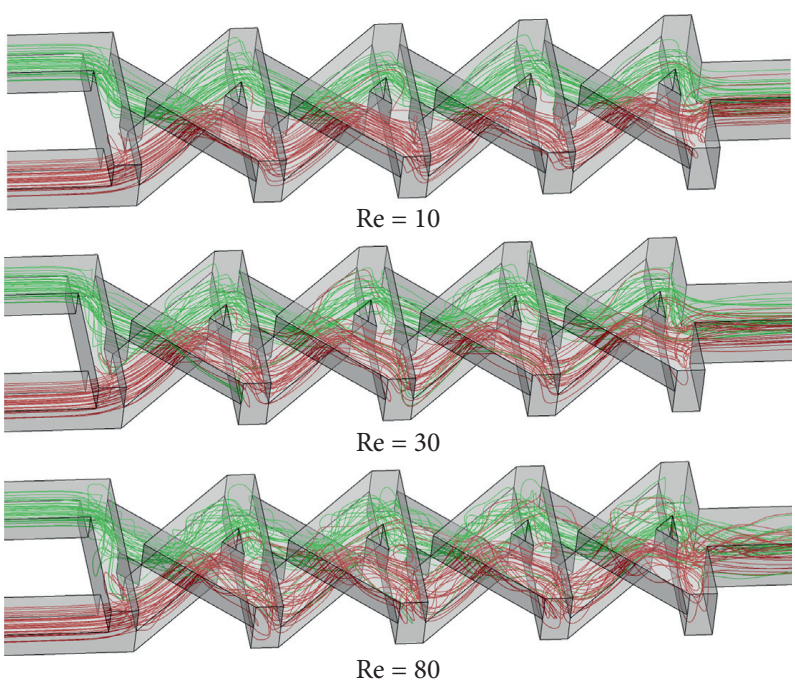

Figure 7: Streamlines distribution invented from inlets (inlet 1 and inlet 2) for the proposed micromixer for $\operatorname{Re}=0.2,10,30,60$, and 80 .

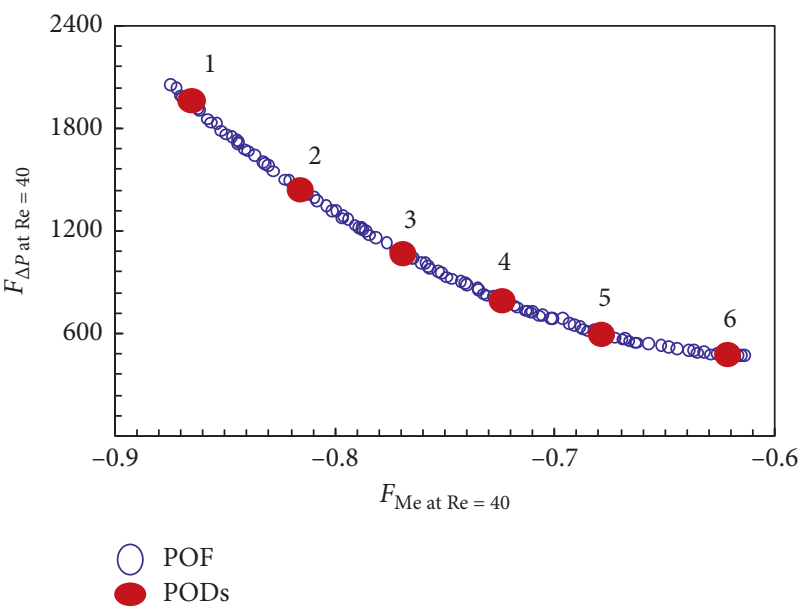

FIgURE 8: Pareto-optimal front for mixing enhancement and overall pressure loss at $\operatorname{Re}=40(\mathrm{FME}$ at $\operatorname{Re}=40 \mathrm{vs} . \mathrm{F} \Delta \mathrm{P}$ at $\mathrm{Re}=40)$.

TABLE 3: Objective function values at six different PODs (mixing enhancement at the exit and pressure drop at $\mathrm{Re}=40$ ).

\begin{tabular}{llllcccccc}
\hline \multirow{2}{*}{ PODs } & \multicolumn{2}{c}{ Design variables } & \multicolumn{2}{c}{ Surrogate prediction } & \multicolumn{2}{c}{ Numerical analysis } & \multicolumn{2}{c}{$\%$ error } \\
& $H / P$ & $w / P$ & $d / P$ & Mixing enhancement & Pressure drop (Pa) & $M_{o}$ & Pressure drop (Pa) & $M_{o}$ & Pressure drop (Pa) \\
\hline 1 & 1.78 & 0.33 & 0.21 & 0.86 & 1946.5 & 0.83 & 1512.00 & 3.61 & 28.74 \\
2 & 1.74 & 0.39 & 0.22 & 0.82 & 1436.2 & 0.79 & 1034.50 & 3.80 & 38.83 \\
3 & 1.75 & 0.42 & 0.21 & 0.77 & 1078.0 & 0.70 & 781.33 & 10.00 & 37.97 \\
4 & 1.76 & 0.41 & 0.20 & 0.72 & 793.2 & 0.66 & 577.25 & 9.09 & 37.41 \\
5 & 1.72 & 0.38 & 0.22 & 0.68 & 603.6 & 0.62 & 487.01 & 9.68 & 23.94 \\
6 & 1.73 & 0.33 & 0.19 & 0.62 & 477.3 & 0.65 & 382.2 & -4.62 & 24.88 \\
\hline
\end{tabular}

the micromixer with POD-6 represents couple of ovalshaped vortices (reverse revolving) reallocated to the right wall; therefore, the strength of velocity vectors become comparatively weaker.

The velocity vector for the micromixer with POD-1 indicates the strong crosswise sample fluid flow mechanism, and vectors are almost equally spread to the plotted plane.
The strongest crosswise fluid flow mechanism makes the variation in performance of mixing for the micromixer with POD-1 as compared to the micromixer with POD-6. Figure 9(b) represents the vorticity distributions (local), captured for the micromixer with POD-1 and POD-6 on the $y-z$ plane. The vorticity was mathematically calculated using the following method: 

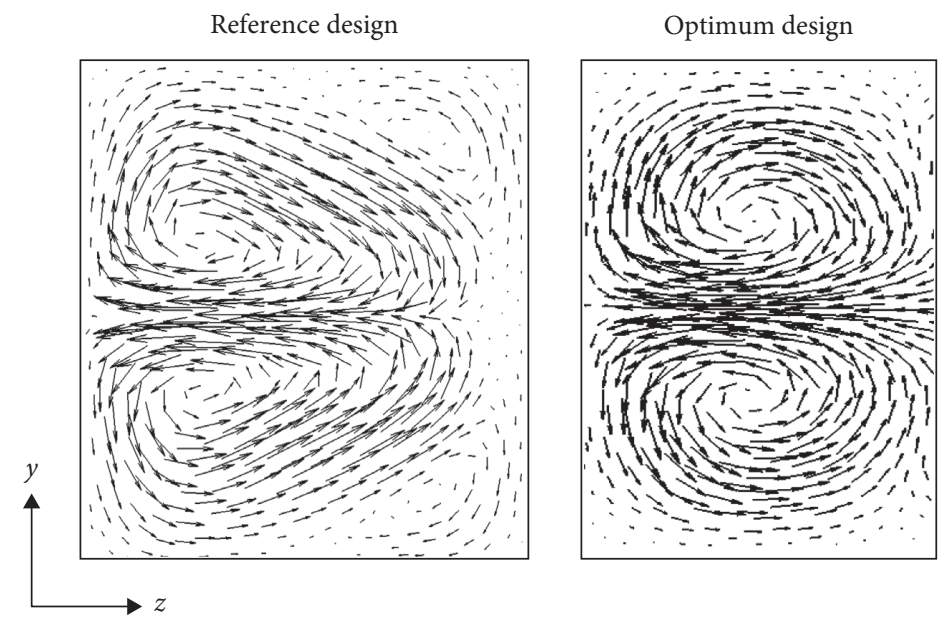

(a)
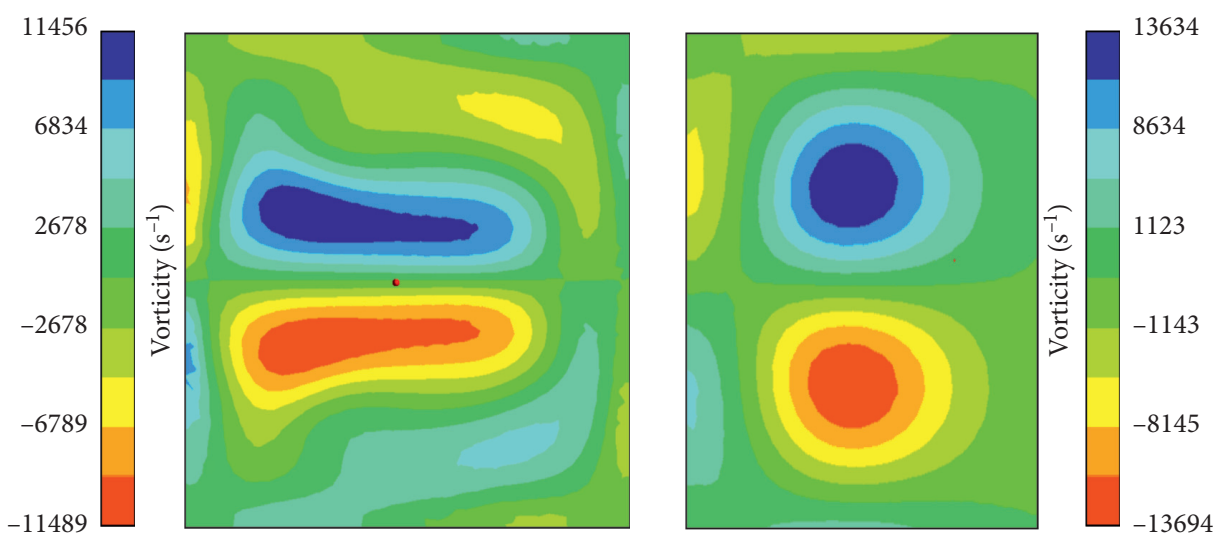

(b)

FIgURE 9: Evaluation of optimum designs and reference design at $\operatorname{Re}=40$. (a) Plot of the velocity vectors. (b) Distributions of the local vorticity.

$$
\omega_{x}=\left(\frac{\partial v_{z}}{\partial y}-\frac{\partial v_{y}}{\partial z}\right)
$$

where $\omega_{x}$ is the vorticity through $x$-direction, $w$ is the velocity component along $z$ direction, and $v$ is the velocity component along $y$ direction. The development of normalized circulation for the micromixer with POD-1 and POD-6 is shown in Figure 9(b).

\section{Conclusions}

Optimal structure of the micromixer with a two-layer serpentine crossing device was accomplished by a multiobjective genetic algorithm and surrogate modeling based on a Navier-Stokes analysis using the trade-off objective functions behavior. The optimization analysis was conducted with three design parameters, i.e., channel width to the pitch span $(w / P)$ ratio, major channel width to the pitch span $(H / P)$ ratio, and channel depth to the pitch span $(d / P)$ ratio. Two objective functions (i.e., mixing index and pressure drop) with trade-off characteristics have been used to solve the multiobjective optimization problem. The contradictory behaviors of the objective functions have been explained through concave POF. Thus, the multiobjective optimization system demonstrates enhancement in the mixing index leaned to rise in the overall pressure loss and vice versa. The numerical data of the thirtytwo design points were used to create the surrogate model; among different surrogate models, in this study, the Kriging metamodel has been used. Results show, as contrast to POD-6, the POD-1 signifies 38\% comparative improvement in the mixing performance, with $307.4 \%$ improvement in the overall pressure loss. The study also concludes a good match (between $3.66 \%$ and $10.0 \%$ ) connecting the numerical analysis values and surrogate predicted values.

\section{Data Availability}

The data used to support the findings of this study are available from the corresponding author upon request.

\section{Conflicts of Interest}

The authors declare that there are no conflicts of interest. 


\section{Acknowledgments}

This research was supported by (EO210005) the Korea Institute of Industrial Technology (KITECH). The authors greatly acknowledge the generous support. Authors greatly acknowledged the support of the students of Industrial and Production Engineering Department in Jashore University of Science and Technology. Specifically, S.M. Sayeed Anwar, Al Sani, Faisal Mina, and Arafat Sarkar.

\section{References}

[1] W. Ehrfeld, V. Hessel, and H. Lowe, Microreactors: New Technology for Modern Chemistry, Vol. 1, Wiley VCH, Weinheim, Germany, 2000.

[2] K. Benz, K.-P. Regenauer, J. Schiewe et al., "Utilization of micromixers for extraction processes," Chemical Engineering \& Technology, vol. 24, no. 1, pp. 11-17, 2001.

[3] V. Hessel and H. Löwe, "Micromixers-a review on passive and active mixing principles," Chemical Engineering Science, vol. 60, no. 8, pp. 2479-2501, 2005.

[4] N. T. Nguyen and Z. Wu, "Micromixers-a review," Journal of Micromechanics and Microengineering, vol. 15, no. 2, pp. 1-16, 2005.

[5] O. Byrde and M. L. Sawley, "Optimization of a Kenics static mixer for non-creeping flow conditions," Chemical Engineering Journal, vol. 72, no. 2, pp. 163-169, 1999.

[6] F. Schönfeld, V. Hessel, and C. Hofmann, "an optimised splitand-recombine micro-mixer with uniform "chaotic" mixing," Lab Chip, vol. 4, no. 1, pp. 65-69, 2004.

[7] Y. Du, Z. Zhang, C. Yim, M. Lin, and X. Cao, "A simplified design of the staggered herringbone micromixer for practical applications," Biomicrofluidics, vol. 4, no. 2, Article ID 024105, 2010.

[8] J. Aubin, D. F. Fletcher, J. Bertrand, and C. Xuereb, "Characterization of the mixing quality in micromixers," Chemical Engineering \& Technology, vol. 26, no. 12, pp. 1262-1270, 2003.

[9] T. J. Johnson and L. E. Locascio, "Characterization and optimization of slanted well designs for microfluidic mixing under electroosmotic flow," Lab on a Chip, vol. 2, no. 135, pp. 135-40, 2002.

[10] H. Aref, "Stirring by chaotic advection," Journal of Fluid Mechanics, vol. 143, no. 1-2, 1984.

[11] J. M. Ottino, The Kinematics of Mixing: Stretching, Chaos, and Transport, Cambridge University Press, Cambridge, UK, 1989.

[12] R. H. Liu, M. A. Stremler, K. V. Sharp et al., "Passive mixing in a three-dimensional serpentine microchannel," Journal of Microelectromechanical Systems, vol. 9, no. 2, pp. 190-197, 2000.

[13] A. D. Stroock and S. K. Dertinger, "Chaotic mixer for microchannels," Science, vol. 295, no. 5555, pp. 647-651, 2002.

[14] D. S. Kim, S. W. Lee, T. H. Kwon, and S. S. Lee, "A barrier embedded chaotic micromixer," Journal of Micromechanics and Microengineering, vol. 14, no. 6, pp. 798-805, 2004.

[15] L. Wang and J.-T. Yang, "An overlapping crisscross micromixer using chaotic mixing principles," Journal of Micromechanics and Microengineering, vol. 16, no. 12, pp. 2684-2691, 2006.

[16] H. M. Xia, S. Y. M. Wan, C. Shu, and Y. T. Chew, "Chaotic micromixers using two-layer crossing channels to exhibit fast mixing at low Reynolds numbers," Lab on a Chip, vol. 5, no. 7, pp. 748-755, 2005.

[17] R. Mohebbi, A. A. Delouei, A. Jamali, M. Izadi, and A. A. Mohamad, "Pore-scale simulation of non-Newtonian power-law fluid flow and forced convection in partially porous media: thermal lattice Boltzmann method," Physica A: Statistical Mechanics and Its Applications, vol. 525, pp. 642656, 2019.

[18] B. Afra, A. A. Delouei, M. Mostafavi, and A. Tarokh, "Fluidstructure interaction for the flexible filament's propulsion hanging in the free stream," Journal of Molecular Liquids, vol. 323, Article ID 11941, 2021.

[19] A. Amiri Delouei, M. Nazari, M. H. Kayhani, S. K. Kang, and S. Succi, "Non-Newtonian particulate flow simulation: a direct-forcing immersed boundary-lattice Boltzmann approach," Physica A: Statistical Mechanics and Its Applications, vol. 447, pp. 1-20, 2016.

[20] M. A. Ansari and K. Y. Kim, "Application of the radial basis neural network to optimization of a micromixer," Chemical Engineering Technology, vol. 30, no. 7, pp. 962-966, 2007.

[21] N. S. Lynn and D. S. Dandy, "Geometrical optimization of helical flow in grooved micromixers," Lab on a Chip, vol. 7, no. 5, pp. 580-587, 2007.

[22] S. Hossain, M. A. Ansari, A. Husain, and K.-Y. Kim, "Analysis and optimization of a micromixer with a modified Tesla structure," Chemical Engineering Journal, vol. 158, no. 2, pp. $305-314,2010$.

[23] C. A. Cortes-Quiroz and A. Azarbadegan, "An efficient passive planar micromixer with fin-shaped baffles in the tee channel for wide Reynolds number flow range," World Academic Science Engineering and Technology, vol. 61, pp. 170175, 2010.

[24] S. Hossain, A. Husain, and K.-Y. Kim, "Optimization of micromixer with staggered herringbone grooves on top and bottom walls," Engineering Applications of Computational Fluid Mechanics, vol. 5, no. 4, pp. 506-516, 2011.

[25] A. Afzal and K. Y. Kim, "Multi-objective optimization of a micromixer with convergent-divergent sinusoidal walls," Chemical Engineering Journal, vol. 202, pp. 1324-1334, 2014.

[26] A. Afzal and K. Y. Kim, "Multi-objective optimization of a passive micromixer based on periodic variation of velocity profile," Chemical Engineering Communication, vol. 202, pp. 322-331, 2015.

[27] F. Ahmed and S. Hossain, "Numerical and experimental study on mixing in chaotic micromixers with crossing structures," Chemical Engineering Technology, vol. 43, pp. 1866-1875, 2020.

[28] CFX-15.0, Solver Theory, ANSYS Inc., Canonsburg, PA, USA, 2013.

[29] S. Hardt and F. Schonfeld, "Laminar mixing in different interdigital micromixers: II: numerical simulations," AICHE Journal, vol. 49, no. 3, pp. 578-584, 2003.

[30] R. B. Bird and W. E. Stewart, Transport Phenomenon, Wiley, New York, NY, USA, 1960.

[31] C. K. Chung, T. R. Shih, T. C. Chen, and B. H. Wu, "Mixing behavior of the rhombic micromixers over a wide Reynolds number range using Taguchi method and 3D numerical simulations," Biomedical Microdevices, vol. 10, no. 5, pp. 739-748, 2008.

[32] P. Hinsmann, J. Frank, P. Svasek, M. Harasek, and B. Lendl, "Design, simulation and application of a new micromixing device for time resolved infrared spectroscopy of chemical reactions in solution," Lab on a Chip, vol. 1, no. 1, pp. 16-21, 2001. 
[33] M. A. Ansari, K.-Y. Kim, K. Anwar, and S. M. Kim, "A novel passive micromixer based on unbalanced splits and collisions of fluid streams," Journal of Micromechanics and Microengineering, vol. 20, no. 5, Article ID 055007, 2010.

[34] B. J. Kirby, Micro- and Nanoscale Fluid Mechanics: Transport in Microfluidic Devices, Cambridge University Press, Cambridge, UK, 2010.

[35] Matlab, The Language of Technical Computing, The Math Works, Inc., Natick, MA, USA, 2008.

[36] K. Deb, "Multi-objective optimization using evolutionary algorithms," Applied Mathematics in Science, pp. 1-518, Wiley \& Sons, Hoboken, NJ, USA, 2001.

[37] J. D. Martin and T. W. Simpson, "Use of Kriging models to approximate deterministic computer models," AIAA Journal, vol. 43, no. 4, pp. 853-863, 2012.

[38] S. Hossain, N. T. Tayeb, F. Islam et al., "Enhancement of mixing performance of two-layer crossing micromixer through surrogate-based optimization," Micromachines, vol. 12, no. 2, p. 211, 2021. 